\title{
Potential Solutions to the Challenges of Low Inertia Power Systems with a Case Study Concerning Synchronous Condensers
}

\author{
M. Nedd \\ University of Strathclyde, UK. \\ marcel.nedd@strath.ac.uk
}

\author{
C. Booth \\ University of Strathclyde, UK. \\ campbell.booth@strath.ac.uk
}

\author{
K. Bell \\ University of Strathclyde, UK. \\ keith.bell@strath.ac.uk
}

\begin{abstract}
This paper will review the recent and on-going changes to the power system in Great Britain (GB). One of the main challenges resulting from these changes in generation mix is the assurance of frequency stability in a low inertia system, and the provision of adequate dynamic responses to frequency changes, while meeting the requirements of the energy trilemma. Specifically, the increase in penetration of non-synchronous generation increases the risk of undesired operation of protection devices and contributes to a shortage of dynamic immediate response to frequency changes.

A range of potential solutions will be briefly reviewed in this paper including, demand side response (DSR), energy storage, synthetic inertia, and synchronous condensers. A case study concerned with evaluating the impact that synchronous compensation may have in a low inertia power system will be described in the paper. The paper will conclude with an outline of the avenues for further study towards addressing the challenge of frequency stability and system inertia in a future power system.
\end{abstract}

Index Terms--Demand side response, energy storage, low inertia system, power system protection, RoCoF, synchronous compensation, synthetic inertia.

\section{INTRODUCTION}

The energy network is required to be more sustainable, while maintaining security of supply and availability, without incurring heavy costs to the consumer; such a network will need to be robust enough to support the expected growth in demand amidst the ongoing changes to the energy landscape [1] - [3]. In the United Kingdom (UK), the 2009 Renewable Energy Directive target is to achieve $15 \%$ energy consumption from renewable sources by 2020 , which results in an expectation of $30 \%$ of electricity generation from renewable sources [4]. Furthermore, a 2050 target of at least $80 \%$ reduction in GHG emissions compared to 1990 baseline levels is put forward by [5]. The resultant impact is the increased proliferation of low carbon, particularly renewable, generation. In Great Britain (GB), the two major renewable sources are wind and solar power, which are (in their majority) converter-connected technologies and their percentage share of generation is expected to grow [3]. Following the fifth carbon budget legislated in July 2016, the power sector is expected to increase the low carbon generation percentage (including renewables) from $45 \%$ to $80 \%$ by 2030 , while coal plants are expected to close, and system flexibility increased via interconnection, demand side response, storage, and flexible back-up capacity [6].

The increasing penetration of non-synchronous technologies (solar, wind and interconnectors) on the transmission network presents challenges [7]. Traditionally, transmission connected synchronous machines have been the main source of system inertia, since they are inherently electromagnetically coupled to the transmission network. Conversely since non-synchronous generation technologies are connected to the transmission network, often via a solidstate electronic converter, they are decoupled, and therefore do not have the same inherent capability of providing inertia to the power system [8], [9].

The inertia of a power system is an inherent capability that affects the rate of change of frequency (RoCoF) following a system event [9], [10]. The relationship between system inertia and RoCoF is illustrated via the swing equation, shown in (1) below where $\mathrm{dP}$ is the change in active power, $\mathrm{H}_{\text {sys }}$ is the system inertia, $\mathrm{f}_{\mathrm{o}}$ is the system frequency, $\mathrm{df}$ is the change in frequency over time, dt. As system inertia reduces, the RoCoF $(d f / d t)$ increases.

$$
d P=\left(\frac{2 \times H_{s y s}}{f_{o}}\right) \times\left(\frac{d f}{d t}\right)
$$

Generally, a power system with larger system inertia will be more resilient to frequency disturbances than a power system with smaller system inertia [1], [11], [12]. If the RoCoF following a frequency deviation is too high, it increases the risk of cascading frequency events, as a result of the tripping of RoCoF relays. RoCoF relays are widely used in some countries, including the UK and Ireland, in loss of mains (LOM) protection for distributed generation [13], [14]. These relays are designed to open the circuit when the system RoCoF reaches a given limit [15]. The resultant impact of the undesirable operation of ROCoF relays in low inertia power systems is an increased risk of loss of supply. Consider the recent Australian power incident, which illustrates the concern [16] - [18]. In this instance, the Australian power network experienced a frequency collapse in the presence of a high penetration of wind and inadequate provision of reserve 
to secure against disturbances that might have been reasonably expected, albeit exacerbated by undocumented features of some wind turbines' protection systems.

The increase in penetration of converter-connected devices is not limited to generation but is also expected to be the case with demand [19], reducing the inertia that demand provides the power system; while the changing nature of demand, i.e., the increased penetration of constant power loads [20], [21], is expected to lead to a reduction of active power response to frequency deviations.

The future energy scenarios (FES), published annually by National Grid (the GB system operator), put forward four broad scenarios of the future GB power network that encapsulate the ideas behind a changing energy landscape. These scenarios are: gone green, slow progression, consumer power and no progression - details behind these scenarios can be found in [19], [22], [23]. The scenarios and results of accompanying studies form the basis for other documents from the GB system operator ( $\mathrm{SO}$ ); most notably the system operability framework (SOF) reports, which present the challenges of a future power system alongside discussions around potential solutions and avenues that require further investigation [7], [24].

As put forward by the recent SOF 2016 report and agreed upon by industry experts, one of the main challenges resulting from these changes to the electrical power landscape is the assurance of frequency stability in a low inertia system, and the provision of adequate dynamic responses to frequency changes, while meeting the requirements of the energy trilemma. This challenge relates to the behaviour of the system during the first $30 \mathrm{~s}$ following a frequency event, in terms of RoCoF and frequency deviations, which are collectively determined by the level of system inertia, the size of generation - demand imbalance, and the magnitude and speed of delivery of frequency response.

There are operational limits, relating to both RoCoF and minimum or maximum frequency excursions that the SO must adhere to in the event of a disturbance. In a system with decreasing levels of system inertia the challenge of complying with these limits increases, leading to a need for the consideration of potential solutions. This is coupled with the knowledge that the increase in penetration of nonsynchronous generation raises the risk of undesired operation of protection devices and further contributes to the need for more adequate dynamic responses to frequency changes.

Ultimately, the concern regards the integrity of low inertia power systems with a high penetration of non-synchronous sources of power and converter-interfaced loads. Some of the systems that are already experiencing operational scenarios in which credible loss of in-feed events within normal security standards give rise to concern include those on the island of Ireland and in GB.

Some potential solutions to the challenge of low inertia power system will be introduced in Section II. A case study concerned with evaluating the impact that synchronous compensators may have in a low inertia power system will be presented in Section III. The paper will conclude with an outline of the avenues for further study towards addressing the challenge of frequency stability and system inertia in a future power system.

\section{Potential Solutions}

One potential solution, in the GB context, is to raise the RoCoF limit at the relevant relays or to remove the RoCoF limits altogether; doing so will minimise the risk of undesirable operation of RoCoF relays.

In GB, the RoCoF limit has been changed to $1 \mathrm{~Hz} / \mathrm{s}$ for all new and existing generators with a delay of $500 \mathrm{~ms}$, while synchronous generators commissioned before 1st July 2016 can have a minimum setting of $0.5 \mathrm{~Hz} / \mathrm{s}$ with the same delay [25]. The original document gave existing synchronous and non-synchronous generators until the 1st July 2016 to make the relevant changes; however, coordinating and implementing these changes, particularly in reference to distributed generation has proven challenging, thus the $6 \mathrm{GW}$ of distributed generation that is still operating with RoCoF relays setting of $0.125 \mathrm{~Hz} / \mathrm{s}$. This leads to a practical RoCoF limit of $0.125 \mathrm{~Hz} / \mathrm{s}$.

One way to address this practical limit is to constrain the largest loss of demand or generation (largest loss risk), to reduce the risk of a cascading event because of the tripping of LOM RoCoF relays [7]. Given the RoCoF limit and system inertia, the largest loss risk can be calculated using the swing equation in (1). For instance, a system with post-fault inertia of 130 GVAs has a largest loss risk of $650 \mathrm{MW}$, which in terms of generation requires curtailment of any single unit (generator or interconnector) supplying power at the normal loss limit. The normal loss limit is the loss of a maximum of 1 $\mathrm{GW}$, such that frequency deviates no more than $0.5 \mathrm{~Hz}$ from nominal $(50 \mathrm{~Hz})$. It can be inferred that there are two factors determining the largest loss risk, the size of frequency deviation and RoCoF. Consequently, the system must be secured against the lower of the two limits, i.e., in the case of a system with 130 GVAs of inertia, the RoCoF limit results in a smaller amount of permitted loss than the normal loss limit, and the system must be secure against that limit. Alternatively, raising the inertia of the power system, using solutions such as synchronous compensators, can also reduce the risk of undesirable operation of RoCoF relays in low inertia power systems. The impact of deploying additional synchronous compensation in a low inertia power system will be investigated in Section III.

There are other solutions being considered in response to some of the challenges posed by a low inertia power system. These include (but are not limited to), demand side response, energy storage, and synthetic inertia.

\section{A. Demand Side Response}

Demand side response (DSR) is a technique that involves managing the power demand from the consumer's side of the power flow. DSR aids in addressing some of the problems of frequency containment arising from generation - load 
imbalance, via the provision of active power response from demand [26]. In a typical DSR scheme, proactively participating consumers (typically under a prearranged agreement) reduce their demand in order to compensate for the effective loss of generation. DSR has been considered in GB and trials have been conducted to see how effective it will be at alleviating the grid constraints, towards being included as a tool in the future smart grid [26] - [29].

DSR requires consumer participation, communication, and data management, and together with other solutions, it can be a viable source of the provision of response; however, the costs for system-wide deployment are not unsubstantial and proactive participation presents its own constraints. DSR is a potentially viable solution to frequency containment and RoCoF issues [2], [26].

\section{B. Energy Storage}

Thermal storage, pumped hydro storage, compressed air, fly wheel, battery, and even hydrogen, are optional methods for energy storage being developed, which when applied to the grid will have aid in addressing the challenge of frequency stability in low inertia power systems [26], [30], [31]. These technologies allow excess energy produced to be stored for later use. Energy storage can be directly incorporated into frequency response services and activated very quickly, slowing down the RoCoF during a frequency event. Energy storage can also be applied to technologies like wind and solar, where generation can be considered variable; energy can be stored during overproduction and utilized during underproduction [26], [30 - 32].

Many storage technologies are considered expensive solutions that require further development [31], with concerns in relation to detecting when (and how much) response is needed. This concern is addressed, in part, by the SO's Smart Frequency project, which puts forward a method and system for coordinating the active power response of a range of providers across locational boundaries [8], [33].

Limiting factors aside, this year's accepted tenders for the SO's enhanced frequency response (EFR) service are all via energy storage, providing a total of $200 \mathrm{MW}$ of response that can be fully delivered in $1 \mathrm{~s}$ or less [34], with a few hundred milliseconds activation time; which in comparison to similar scenarios without EFR, will reduce total active power response requirement, while giving other response providers time to act by slowing down the RoCoF.

\section{Synthetic Inertia}

Synthetic inertia is the provision of an active power response that mimics the active power response that is provided by synchronous inertia (the inherent inertia of synchronous machines). Synthetic inertia in wind turbines employs the use of a controller to demand more torque from the turbine, while feeding its controller a false rise in rotor speed to overcome the actual fall in rotor speed because of extracting more torque; however, this method comes with an energy deficit a few seconds later, known as the 'recovery period' [35].
During a generation - load imbalance, the rate and magnitude of frequency deviation can be dampened via an injection of power, thereby aiding to preserve the integrity of the power; however, unlike traditional thermal plants, the wind that a wind farm exploits cannot be controlled. In the case of a loss of in-feed (LOIF) event, an increase in wind farm power production to compensate via synthetic inertia alone raises inherit questions about this method as a complete solution to the provision of adequate dynamic responses to frequency changes [11], [26], [36], [37]. Another question raised by industry experts about synthetic inertia is the inherent delay in its inertial response, unlike the inertial response of a synchronous machine, likening synthetic inertia to very fast acting active power responses. Notwithstanding, when considering services such as EFR, there is a benefit in having very fast acting response; the challenge with this method is in its inherent recovery period. However, combined with other frequency response solutions, synthetic inertia may prove valuable.

\section{SYNCHRONOUS COMPENSATORS}

A synchronous compensator (SC), also known as a synchronous condenser, is an inherently unloaded synchronous motor that is considered to have the potential to offer, among other benefits, a boost to system inertia and an increase to system fault level [38]. In [26], SCs are considered to have the potential to solve RoCoF issues, regional stability, voltage dips and management, and HVDC commutation. It is an established technology, which could be purchased for purpose or retrofitted by taking advantage of thermal plants scheduled for decommissioning, and saving on the implementation costs; however, it will require further investigation and market development before GB wide deployment (see [39]).

Using in-house developed GB transmission models and operating algorithms, a study was conducted to investigate the potential impacts that synchronous compensation (SC) would have on RoCoF and fault levels.

\section{RoCoF Study}

Fig. 1 below illustrates the impact of deploying a 5 GVA $\mathrm{SC}$ with a 2-s inertia constant, where a comparison is made in terms of RoCoF for scenarios with and without the SC. The study was conducted for a 75 GVAs scenario with $20 \mathrm{GW}$ demand, and it indicates that a 5 GVA SC, while considering dynamic system elements, can reduce the RoCoF from 0.116 $\mathrm{Hz} / \mathrm{s}$ to $0.103 \mathrm{~Hz} / \mathrm{s}$ for a $375 \mathrm{MW}$ LOIF. Similarly, it was also observed that the deployment of a 5 GVA SC with a RoCoF limit of $0.125 \mathrm{~Hz} / \mathrm{s}$ raised the LOIF tolerance from $410 \mathrm{MW}$ without the SC, to $460 \mathrm{MW}$ with the SC.

It is observed that the deployment of a 5 GVA SC can permit a larger loss limit for a given RoCoF limit, minimising the need for system constraints (e.g. constraining the largest loss risk) that may be required to secure the system, potentially reducing the costs associated with the provision of system security. Similarly, a system condition that would 
have originally been at the cusp of breaching the RoCoF limit is brought further within acceptable limits when a 5 GVA SC is introduced to the network. This reduction in RoCoF, following a frequency event, allows more time for other services to respond and could contribute to a reduction in the overall active power requirement for frequency containment. Furthermore, a reduction in the RoCoF can mitigate the risk of a cascading event because of the undesired tripping of RoCoF protection applied to distributed generation, which would exacerbate the initial system disturbance.

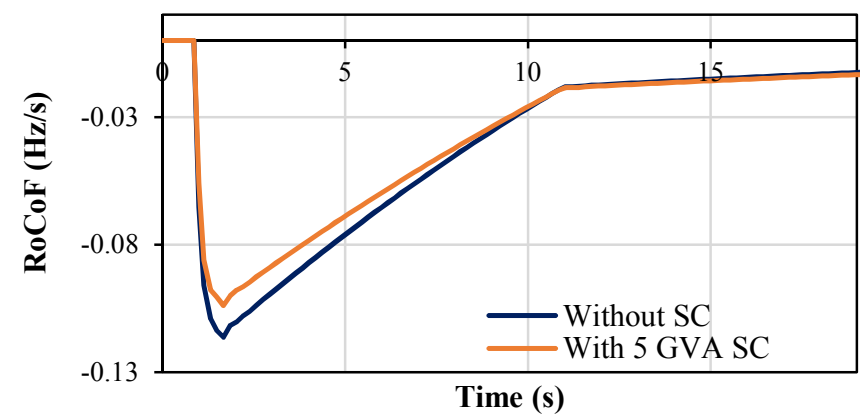

Fig. 1: RoCoF comparison with system dynamics.

\section{E. Fault Level Study}

A study was conducted concerning the benefits that the deployment of a SC would have on fault levels and short circuit ratio (SCR); of particular interest is the potential to avoid/mitigate the risk of loss of commutation on currentsourced converter-based (LCC) HVDC links, e.g. the Western Link project connecting Ayrshire in Scotland to the Wirral in England [40]. This risk is assessed using the short circuit ratio on the AC system at the terminal(s) of the Western link, illustrated in Fig. 2. Where SCRs of less than or equal to 3 are deemed to increase the risk of loss of commutation (in the event of an AC system fault near a converter's terminals) in LCC-HVDC systems, with a SCR of greater than 3 desired to minimise the risk of commutation loss [41].

The study was conducted based on a three-phase busbar fault at Hunterston, illustrated as ' $\mathrm{S} 1$ ' in Fig. 2 below, under current summer minimum demand conditions using DigSILENT's IEC 60909 [42] minimum short circuit tool on PowerFactory; faults levels were recorded and SCR calculated.

A SC, with capacity varied from 0 to 1 GVA in 200 MVA steps, was placed at two locations, Neilston (S2 in Fig. 2) and Longannet (S3 in Fig. 2). Fig. 3 below shows the trends of the impact on fault levels, in terms of apparent power (at $80 \mathrm{~ms}$ after the fault inception) and short circuit ratio at Hunterston with increasing capacities of synchronous compensation at both locations. The study indicates that the fault level and the short circuit ratio at Hunterston rises with increasing capacities of synchronous compensation, effectively strengthening the AC system. Furthermore, the increase in fault levels and short circuit ratio is pronounced if the synchronous compensator is placed electrically closer to Hunterston.

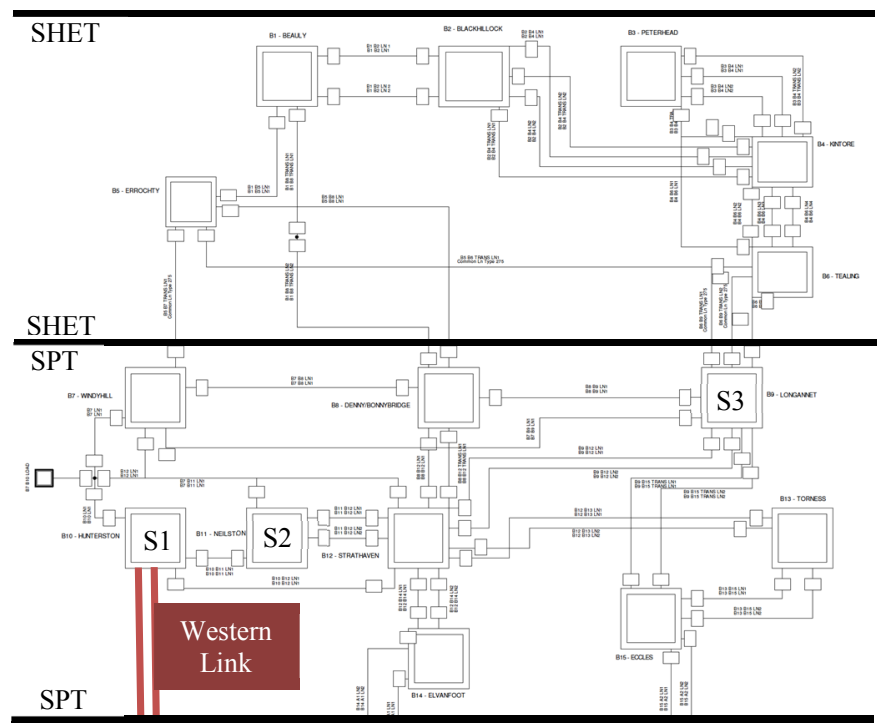

Fig. 2: Sectioned image of the in-house developed GB reduced network model showing Scotland.

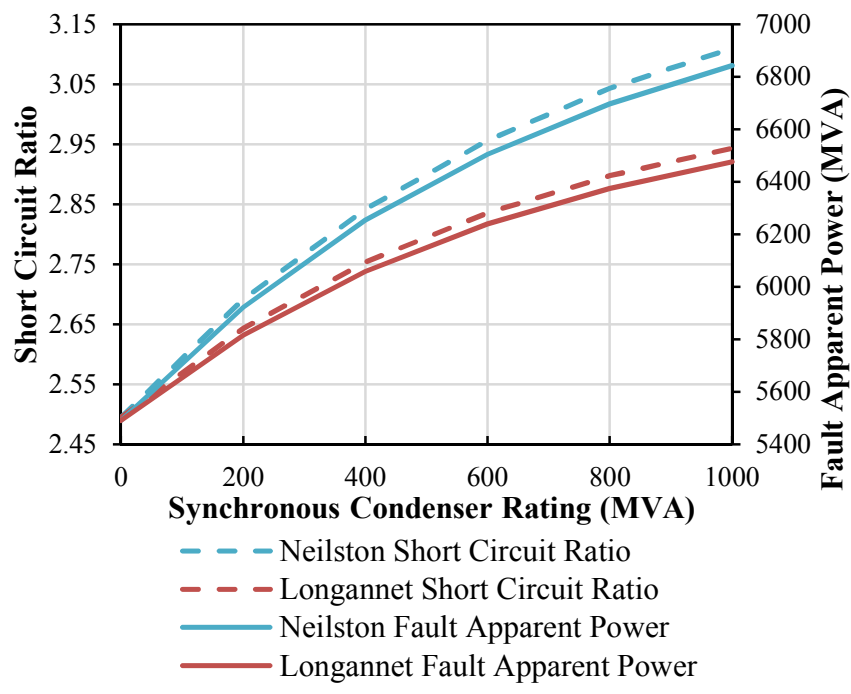

Fig. 3: Fault MVA and short circuit ratio at Hunterston for increasing penetration of synchronous compensation at Neilston and Longannet.

\section{DISCUSSION}

The practical RoCoF limit of $0.125 \mathrm{~Hz} / \mathrm{s}$ presents an immediate challenge that can be remedied by adding more inertia to the power system, constraining the largest loss risk, or deploying the new RoCoF limit across the GB. Currently, during periods of low inertia, the SO constrains the largest loss risk to keep RoCoF within the limit [7]. The SOF report suggests that by $2020 / 21$ at a RoCoF limit of $0.125 \mathrm{~Hz} / \mathrm{s}$, the loss limit will be below $1 \mathrm{GW}$ a little more than half of the time; where in the 2025/26 gone green scenario, the loss limit is below $1 \mathrm{GW}$ for most of the year. The economic impact can be illustrated by considering the following scenario.

If by $2025 / 26$ the loss limit is $700 \mathrm{MW}$ about $60 \%$ of the time then BritNed, a $1 \mathrm{GW}$ interconnector, will have to be curtailed by $300 \mathrm{MW}$ to minimize the risk to system security. If this interconnector is constrained $60 \%$ of the year, a total of 
1576.80 GWh will be curtailed. The Aurora report puts GB market price at $£ 43 / \mathrm{MWh}[43]$; therefore, the curtailed energy would cost at least $£ 67.8 \mathrm{~m}$ over that year. National Grid in [8] puts the cost of this solution at $£ 268 \mathrm{~m}$ per annum by 2020 , expected to increase year by year.

These figures are indicative, meant to illustrate the scale of the cost of constraining the single loss limit. However, it should be noted that this sort of curtailment already takes place (see [7]), and while industry experts believe it is currently a viable option, it is considered an interim solution, as it is expected that in the future the cost will increase, fueled in part by more interconnector capacity [7], [43]. At which point, the cost of curtailing the largest single loss risk could prove too high, potentially eliminating it as a viable solution.

While synchronous compensation provides a marginal benefit to RoCoF, this benefit increases as the system inertia reduces. In addition, the deployment of synchronous compensation provides other benefits aside from frequency stability; for example, the boost to fault levels in a weaker system that also utilizes line commutated converters, as shown in Section III. It will be useful to conduct further studies concerning the location of the synchronous compensation deployed, alongside control strategies that will optimize the performance of the synchronous compensator. In GB, the NIC funded Phoenix project will conduct studies investigating this, alongside other aspects relevant to largescale deployment [44].

DSR and energy storage are potentially useful options that address the requirement of additional dynamic responses; however, their impact on initial RoCoF following a frequency event is minimal at best. Synthetic inertia may provide benefits to the initial RoCoF; however, further study will be required to quantify the impact, as well as the impact of DSR and energy storage on the dynamic response requirements of the system following a frequency event, in terms of frequency containment and restoration. Another avenue for further study is an investigation of the benefits and limitations of DSR, storage, synthetic inertia, and SC, in the context of distributed resources; i.e., a study addressing the impact of these options in terms of where they are placed within the GB power network.

\section{ACKNOWLEDGEMENTS}

This work has been supported through the EPSRC Centre for Doctoral Training in Future Power Networks and Smart Grids (EP/L015471/1) with industry support from ScottishPower Energy Networks.

\section{REFERENCES}

[1] H. Urdal, "Impact on T\&D protection systems from low fault," in Substation Technology 2009: Analysing the Strategic and Practical Issues of Modern Substation, Birmingham, 2009.

[2] European Electricity Grid Initiative (EEGI), "Reasearch and innovation roadmap 2013-2022," 2013.

[3] National Grid, "Electricity Ten Year Statement 2016," 2016.

[4] Department of Energy and Climate Change, "UK Renewable Energy Roadmap Update 2013," 11 2013. [Online]. Available: https://tinyurl.com/pm688sj. [Accessed 0102 2017].

[5] HM Government, "2050 Pathways Analysis," 07 2010. [Online]. Available: https://tinyurl.com/ngjadgj. [Accessed 0102 2017].

[6] Committee on Climate Change, "Meeting Carbon Budgets Implications of Brexit for UK climate policy (Briefing Note)," October 2016. [Online]. Available: https://tinyurl.com/k3clwta. [Accessed 6 May 2017].

[7] National Grid, "System Operability Framework 2016," National Grid, 2016.

[8] National Grid, "Enhanced frequency control capability (EFCC)," 10 2014. [Online]. Available: https://tinyurl.com/h3k4rgw. [Accessed 01 02 2017].

[9] P. Tielens and D. V. Hertem, "The relevance of inertia in power systems," Renewable and Sustainable Energy Reviews, vol. 55, pp. 999-1009, 2016.

[10] P. Kundur, Power System Stability and Control, McGraw-Hill Inc., 1994.

[11] H. Urdal, R. Lerna, J. Zhu, C. Ivanov and A. Dahresobh, "System strength considerations in a converter dominated power system," IET Renewable Power Generation, vol. 9, no. 1, pp. 10 - 17, 2015.

[12] A. Johnson, "Grid code frequency response working group system inertia," 2010. [Online]. Available: https://tinyurl.com/hd3sk8u. [Accessed 0102 2017].

[13] P. Crolla, A. J. Roscoe, A. Dysko and G. M. Burt, "Methodology for testing loss of mains detection algorithms for microgrids and distributed generation using real-time power hardware-inthe-loop based technique," in 8th International Conference on Power Electronics ECCE Asia, The Shilla Jeju, 2011.

[14] A. Dysko, "Loss of Mains Protection," [Online]. Available: http://tinyurl.com/mtt8mop. [Accessed 1105 2017].

[15] A. D. Adrianti, "Risk Assessment Analysis to Find Optimum ROCOF Protection Settings," in 12th IET International Conference on Developments in Power System Protection (DPSP 2014), Copenhagen, 2014.

[16] E. Hunt, "South Australia blackout: entire state left without power after storms," 28 September 2016. [Online]. Available: http://tinyurl.com/zmwatpj. [Accessed 1105 2017].

[17] B. P. Hearda, B. W. Brookb, T. M. L. Wigleya and C. J. A. Bradshawd, "Burden of proof: A comprehensive review of the feasibility of $100 \%$ renewable-electricity systems," Renewable and Sustainable Energy Reviews, vol. 76, pp. 1122 - 1133, 2017.

[18] AEMO, "Australian Energy Market Operator. Update report- Black system event in South Australia on 28 September 2016," 19 October 2016. [Online]. Available: http://tinyurl.com/leyu6ov. [Accessed 1105 2017].

[19] National Grid, "Future Energy Scenarios," 2014.

[20] D. Boroyevich, I. Cvetković, D. Dong, R. Burgos, F. Wang and F. Lee, "Future Electronic Power Distribution Systems: A contemplative view," in 12th International Conference on Optimization of Electrical and Electronic Equipment, Brasov, 2010.

[21] D. Chakravorty, B. Chaudhuri and S. Y. R. Hui, "Estimation of Aggregate Reserve with Point-of-Load Voltage Control," IEEE Transactions on Smart Grid, vol. PP, no. 99, pp. 1 - 10, 25 April 2017.

[22] National Grid, "Future Energy Scenarios," 2015.

[23] National Grid, "Future Energy Scenarios," 07 2016. [Online]. Available: https://tinyurl.com/ofeyeys. [Accessed 2102 2017].

[24] National Grid, "System Operability Framework," 2015.

[25] Ofgem, "Changes to the Distribution Code and Engineering Recommendation G59: Frequency changes during large disturbances and their impact on the total system," 2307 2014. [Online]. Available: https://www.ofgem.gov.uk/sites/default/files/docs/2014/07/gc0035_aut hority_decision_0.pdf. [Accessed 0312 2015].

[26] National Grid, "Electricity Ten Year Statement (2014)," [Online]. Available: https://tinyurl.com/jz2gzqk. [Accessed 1105 2017].

[27] E. Kuriakose, "Enhancement of power system stability by optimal 
adaptive under frequency load shedding using artificial neural networks," International Journal of Advanced Research in Electrical Electronics and Instrumentation Engineering, vol. 2, no. 1, pp. 12 - 20 , 2013.

[28] A. Saffarian and M. Sanaye-Pasand, "Enhancement of power system stability using adaptive combinational load shedding methods," IEEE Transactions on Power Systems, vol. 26, no. 3, pp. 1010 - 1020, 2011.

[29] P. Ajay-D-Vimal and R. M. Sudhakaran, "Optimal load shedding in power system strategies with voltage stability indicators," Engineering, vol. 2, no. 1, pp. 12 - 21, 2010.

[30] P. F. Ribeiro and M. L. Crow, "Energy storage systems for advanced power applications," Proceedings of the IEEE, vol. 89, no. 12, pp. 1744 - 1756, 2001 .

[31] D. Bazargan, S. Filizadeh and A. M. Gole, "Stability analysis of converter-connected battery energy storage systems in the grid," IEEE Transactions on Sustainable Energy, vol. 5, no. 4, pp. 1204 - 1212, 2014.

[32] J. Zeng, B. Zhang, C. Mao and Y. Wang, "Use of battery energy storage system to improve the power quality and stability of wind farms," International Conference on Power SYstem Technology, 2006.

[33] N. Grid, "Smart Frequency Control: Project Fact Sheet," [Online]. Available: http://tinyurl.com/mw9wvv2. [Accessed 1105 2017].

[34] National Grid, "Enhanced Frequency Response," [Online]. Available: http://tinyurl.com/h374htw. [Accessed 1105 2017].

[35] Supergen Wind, "Supergen Wind Consortium 6th Educational Seminar: Synthetic Inertia," September 2011. [Online]. Available: http://tinyurl.com/mt6f37d. [Accessed 1105 2017].

[36] F. Gonzalez-Longatt, "Impact of synthetic inertia from wind power on the protection/control schemes of future power systems: simulation study," in 11th Inernational Conference on Developments in Power Systems Protection, Birmingham, 2012.

[37] F. Gonzalez-Longatt, E. Chikuni, W. Stemmet and K. Folly, "Effects of the synthetic inertia from wind power on the total system inertia after a frequency disturbance," in IEEE PES Power Africa 2012 Conference and Exposition, Johannesburg, 2012.

[38] N. H. Thi, Y. Guangya, A. H. Nielsen and P. H. Jensen, "Frequency stability improvement of low inertia systems using synchronous condensers," in 2016 IEEE International Conference on Smart Grid Communications, 2016.

[39] Ofgem, "Electricity Network Innovation Competition: 2016 Funding Decision," 3011 2016. [Online]. Available: https://tinyurl.com/hk29g4j. [Accessed 2302 2017].

[40] W. Link, Western Link Project, 2017.

[41] A. Grid, Connecting to the future, Levallois-Perret Cedex, 2010.

[42] P. Wall, N. Shams, V. Terzija, V. Hamidi, C. Grant, D. Wilson and e. al., "Smart frequency control for the future GB power system," in 2016 IEEE PES Innovative Smart Grid Technologies Conference Europe (ISGT-Europe), 2016.

[43] Aurora, "Dash for interconnection," 02 2016. [Online]. Available: https://tinyurl.com/z44hsvh. [Accessed 2002 2017].

[44] Ofgem, "Electricity NIC submission: Scottish Power Transmission Phoenix," 3011 2016. [Online]. Available: https://tinyurl.com/n96hx9v. [Accessed 1505 2017]. 